IOS Press

\title{
Patients in need of medicine information
}

\author{
I. Kazaryan ${ }^{\mathrm{a}}$ and A. Sevikyan ${ }^{\mathrm{b}, *}$ \\ ${ }^{\mathrm{a} D r u g}$ Utilization Research Group PO, Yerevan, Armenia \\ bharmaceutical Management, Yerevan State Medical University, Department of Pharmacy \\ Management, Yerevan, Armenia
}

*Corresponding author. E-mail: sevikyan@mail.ru

BACKGROUNDS: Reliable medicine information is important not only for physicians and pharmacists, but also for patients [6]. However, the results of studies implemented in some countries show that patients may have slightly different needs and preferences in using sources of information [1, $4,5,7]$. The main objective of patient medicines information is assisting consumers to achieve safe and effective use of pharmaceuticals $[2,3]$.

OBJECTIVE: To identify patients' needs in medicine information and sources they use to receive it.

METHODS: We interviewed 1059 people who had visited community pharmacies in 10 regions of Armenia and Yerevan. Previously developed questionnaire was used for interviewing patients. Statistical analysis was conducted using SPSS program.

RESULTS: We found that consumers need medicine information. $68.9 \%$ of respondents often use pharmaceuticals only if necessary medicines information is available. The majority of them believe that it is important to have information about therapeutic indications of pharmaceuticals to be used $(91.8 \%)$, their dosage and method of administration (91.1\%), contraindications (82.4\%), adverse reactions (81.9\%) and the simultaneous use of multiple medicines (76.5\%). 58.9\% of consumers value information about medicine's price. More than $70 \%$ of patients often seek information from health professionals and use medicines package information leaflets (PIL), and more than $75 \%$ of respondents mainly trust the same sources. $71.5 \%$ of respondents read package leaflets, while $42.0 \%$ of consumers do this several times. Only $36.7 \%$ of respondents completely understand information in a leaflet.

CONCLUSIONS: Patients in Armenia need medicine information. They prefer to receive information from sources they trust.

Many patients do not understand the content of package information leaflets (PILs) due to barriers, which can be removed by introducing appropriate regulatory provisions for their content and readability.

Keywords: Patient information leaflet, medicine, information, patient, consumer

\section{References}

[1] Dickinson D, Raynor TDK, Kennedy JG, Bonaccorso S, Sturchio JL. What information do patients need about medicines? BMJ. 2003;327:861-64

[2] EFPIA Position on shortcomings of the Summary of Product Characteristics and the Package Leaflet, and Proposals to Resolve Them. Position paper. European Federation of Pharmaceutical Industries and Associations, Brussels, 2014,5 p.

[3] FIP Statement of policy medicines information for patients. International Pharmaceutical Federation, The Netherlands, 2008, 4 p. 
[4] Fuchs J., Hippius M., Schaefer M. A survey of package inserts use by patients. Hospital Pharmacy Europe 2005;7-8;2931.

[5] Ho C.H., Ko Y., Tan M.L. Patients and sources of drug information in Singapore: Is the Internet replacing former sources? Ann Pharmacother 2009;43(4):732-39.

[6] Jeetu G., Girish T. Prescription Drug Labeling Medication Errors: A Big Deal for Pharmacists. J Young Pharm 2010; 2(1):107-111.

[7] Nair K., Dolovich L., Cassels A., McCormack J., Levine M., Gray J., Mann K., Burns S. What patients want to know about their medications. Canadian Family Physician 2002;1(48):104-10. 